\title{
Reconstruction of the chest wall in locally advanced breast cancer with multi-disciplinary cooperation: a case report of mesh repair plus TRAM combined with DIEP chest wall reconstruction
}

\author{
Shengchao Huang", Pu Qiu\#, Yuanqi Zhang, Jianwen Li, Weizhang Chen, Baoying Chen, Zhu Liang, \\ Zhongzeng Liang, Kangwei Luo, Baoyi Huang \\ Department of Breast Surgery, Oncology, Chest Surgery, Affiliated Hospital of Guangdong Medical University, Zhanjiang, China \\ \#These authors contributed equally to this work. \\ Correspondence to: Yuanqi Zhang; Jianwen Li. Department of Breast Surgery, Oncology, Chest Surgery, Affiliated Hospital of Guangdong Medical \\ University, Zhanjiang 524001, China. Email: 513614508@qq.com; 982688169@qq.com.
}

\begin{abstract}
Locally advanced breast cancer, which is defined as a malignant breast tumor that invades or adheres to the surrounding tissue, is characterized by the invasion of the chest wall and the skin surface by the tumor. Multiple lymph nodes are invaded and fuse into a mass, causing extensive axillary lymph node metastasis. However, locally advanced breast cancer does not exhibit distant metastasis. At present, in most hospitals in China and the rest of the world, this type of breast cancer is primarily managed through systematic and local treatments. However, a consensus concerning the optimal surgical method for chest wall reconstruction, which for many surgeons is a difficult and confusing procedure, has not been reached. In the past, many breast centers had used skin flap combined with hard mesh titanium alloy plate to repair the large chest wall defects. Although titanium alloy plate can maintain the stability of the chest wall, it may have a negative effect on the follow-up radiotherapy of breast cancer patients, which is a controversial method. In addition, titanium alloy mesh also has the risk of deformation and fracture. These factors will cause some hidden dangers to patient safety. According to the research, the soft mesh not only has the characteristics of satisfactory compatibility and robustness for maintaining the stability of chest wall, but also does not affect the postoperative radiotherapy of patients. Combined with the advantages of soft mesh, Our department treated a case of locally advanced breast cancer with chest wall invasion. Through cooperation between the breast surgery and thoracic surgery departments, a mesh repair plus transverse rectus abdominis myocutaneous (TRAM) combined with deep inferior epigastric perforator (DIEP) procedure was performed to remove the breast tumor and repair the large area of skin defect after surgery, and a relatively satisfactory therapeutic effect was achieved. In this case, we took two novel approaches: first, a 4-layer high-density polyethylene mesh was used to repair the defect; secondly, the inferior epigastric artery perforation was anastomosed with the thoracoacromial artery (end-to-end anastomosis) and the inferior epigastric vein perforation was anastomosed with the axillary vein (end-to-side anastomosis).
\end{abstract}

Keywords: Locally advanced breast cancer; transverse rectus abdominis myocutaneous flap (TRAM); deep inferior epigastric perforator (DIEP); mesh repair; multi-disciplinary cooperation; case report

Submitted Feb 20, 2020. Accepted for publication Jun 11, 2020.

doi: $10.21037 /$ gs-20-436

View this article at: http://dx.doi.org/10.21037/gs-20-436

(c) Gland Surgery. All rights reserved. 


\section{Introduction}

As a result of unhealthy lifestyles, psychological pressure, environmental changes and other factors, the number of breast cancer patients increased rapidly, which has seriously affected the health of Chinese women. For women in some less developed areas, a lack of understanding of breast cancer often leads to the development of locally advanced breast cancer. Locally advanced breast cancer usually refers to breast cancer with a primary lesion diameter of $>0.5 \mathrm{~cm}$ or with adhesion and fixation to the skin and chest wall, fusion of regional lymph node metastasis, and ipsilateral supraclavicular lymph node metastasis, but with no distant metastasis. Chest wall repair in these patients poses a problem for many surgeons (1). In the case presented here, a patient with locally advanced breast cancer and chest wall invasion was treated with a mesh repair plus transverse rectus abdominis myocutaneous (TRAM) combined with deep inferior epigastric perforator (DIEP) procedure. In most cases, titanium alloy steel plate is used to repair the defect of chest wall. However, although the titanium plate can maintain the stability of the chest wall, but it affects the subsequent radiotherapy $(2,3)$. In the aspect of perforator vascular anastomosis of flap, the thoracic and thoracoacromial vessel are generally selected as the receiving region. However, once the thoracic vessels are vulnerable to invasion by tumor cells, they cannot be anastomosed with the perforator vessels of the flap. The difference in this case is that a 4-layer highdensity polyethylene mesh was used to repair the defect. In addition, due to the poor condition of thoracic artery and thoracoacromial vein, the inferior epigastric artery perforation was anastomosed with the thoracoacromial artery (end-to-end anastomosis) and the inferior epigastric vein perforation was anastomosed with the axillary vein (end-to-side anastomosis). This method can not only maintain the stability of chest wall, but also does not affect the follow-up radiotherapy. Furthermore, the blood supply of the flap was improved by selecting the thoracic acromial artery and axillary vein as the receiving region. The treatment was delivered through cooperation between the breast surgery and thoracic surgery departments. Using this method, radical resection of the chest tumor and repair of the large skin defect area after surgery achieved a relatively satisfactory therapeutic effect (4). This surgery provided our center with more experience in the repair of the chest wall in locally advanced patients. We present the following case in accordance with the CASE
Report (CARE) guidelines (available at http://dx. doi. org/10. 21037/gs-20-436).

\section{Cases and methods}

\section{Clinical case}

The patient was a 59-year-old female who chiefly complained of a wall mass in the right chest after right breast cancer surgery that had been present for more than 3 months. The right breast had been surgically removed 7 years previously, and a mass with a surface area of approximately 2.0 to $4.0 \mathrm{~cm}^{2}$ had subsequently developed on the right subclavian chest wall. The mass was hard and fixed with a clear boundary and a rough surface (Figure 1).

Medical history: the patient had undergone modified radical mastectomy for right breast cancer in our hospital 7 years prior (the tumor was located in the right breast at the 11 o'clock position and was approximately $2.5 \mathrm{~cm} \times$ $1.3 \mathrm{~cm}$ in size).

In the postoperative pathology 7 years before, cancer metastasis was found in the right axillary lymph nodes (3/11), but no metastasis was found in the group 2 and 3 right axillary lymph nodes $(0 / 7,0 / 1)$. The results of immunohistochemistry were as follows: $\mathrm{ER}(-), \mathrm{PR}(-)$, Her$2(-)$, Ki-67 30\%. The patient was thus diagnosed with invasive ductal carcinoma of the right breast (pT2N1M0 stage II B triple-negative breast cancer). After the operation, the patient was treated with chemotherapy regimens of FEC $\times 3+\mathrm{T} \times 3$, and did not receive radiotherapy.

During the present hospitalization, the patient's examination results were as follows.

Color Doppler ultrasound (breast): a hypoechoic nodule measuring approximately $4.4 \mathrm{~cm} \times 2.0 \mathrm{~cm}$ was visible on the right chest wall, and echoes were uniform.

Chest CT scan: (I) a shadow of irregular soft tissue density was observed next to the second front rib on the right side of the sternum; the maximum dimension was approximately $38 \mathrm{~mm} \times 31 \mathrm{~mm}$, with the mass growing outside of the chest wall and inside of the anocoelia. The mass was considered to be a local recurrence of breast cancer. (II) There were no clear signs of metastasis found in the lungs.

Whole-body bone scan: the blood flow metabolism of the third anterior rib on the right side was abnormally increased.

Enhanced abdominal CT and head MRI: no signs of metastasis were found. 
2010.05 .21

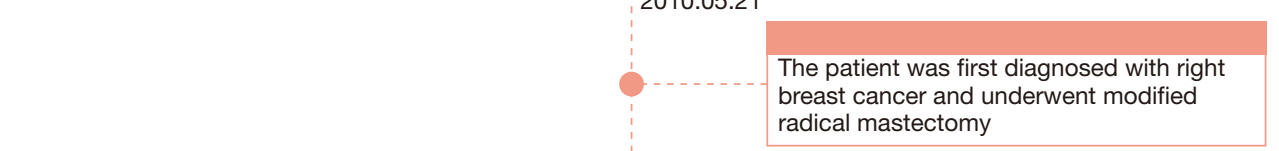

2017.05.31

The patient had a right chest wall mass.

After hospitalization, the pathologic

findings of chest wall tumor were local

recurrence of breast cancer
2017.10.31

The effect of chemotherapy was assessed
on the basis of progressive disease
(PD). Through a multi-disciplinary team
(MDT) discussion, mesh repair plus
TRAM combined with DIEP chest wall
reconstruction was decided upon as the
method of treatment

method of treatment
A first-line chemotherapy strategy for advanced breast cancer, with 4 cycles of rescue chemotherapy of gemcitabine (D1, D8) + cis-platinum (D1, 2, 3), 2 cycles of docetaxel (D1) capecitabine (D1, 14) (TX), and concurrent use of zoledronic acid.

Figure 1 Timeline of patient treatment.

Hollow-needle puncture pathology: combined with the patient's medical history and pathological results 7 years previously, the pathological diagnosis of tumor biopsy was locally recurrent breast cancer.

Immunohistochemistry: ER(-), PR(-), Her-2(-), Ki-67 (30\%).

Preoperative diagnosis: (I) the preoperative diagnosis was recurrence of breast cancer (triple negative) in the right chest wall, and (II) transfer to the third anterior ribs on the right.

After considering the results from the patient's examination and pathology together with the National Comprehensive Cancer network (NCCN) clinical practice guidelines, we applied a first-line chemotherapy strategy for advanced breast cancer, with 4 cycles of rescue chemotherapy of gemcitabine (D1, D8) + cis-platinum (D1, 2, 3), 2 cycles of docetaxel (D1) capecitabine (D1, 14) (TX), and concurrent use of zoledronic acid.

Chest CT reexamination: the density of nodular soft tissue in the second anterior costal-parasternal area of the right side was higher than before, and the size of the nodular soft tissue was approximately $6.4 \mathrm{~cm} \times 5.4 \mathrm{~cm} \times$ $4.4 \mathrm{~cm}$ in size. The lymph nodes adjacent to the soft tissue were $1.6 \mathrm{~cm}$ in diameter and had an unclear boundary. The enhancement scan revealed uneven enhancement, and no clear signs of bone destruction were found in the bone adjacent to the lesion.

The effect of chemotherapy was assessed on the basis of progressive disease (PD). Through a multi-disciplinary team (MDT) discussion, mesh repair plus TRAM combined with DIEP chest wall reconstruction was decided upon as the method of treatment.

Postoperative pathology: (I) the immunohistochemical results combined with the patient's clinical history indicated 
invasive breast adenocarcinoma with focal squamous differentiation. (II) No cancer cells were found in the skin. (III) No cancer metastasis was found in the lymph nodes (right subclavian) (0/4). (IV) No cancer cells were found in the tissue (upper right, lower right, right inner, right lateral margin). The results of immunohistochemistry were as follows: AR (10\%, 1+), ER(-), PR(-), Her-2(2+), ki-67 $(\sim 70 \%)$, FISH(-).

Postoperative diagnosis: the diagnosis was recurrence of breast cancer (triple-negative) in the right chest wall.

Two years after Systemic therapy, we followed up the patient and found no recurrence of tumor or deformation of chest wall. Most importantly, the mesh did not cause symptoms such as: chest wall pain.

\section{Flap design}

Before the operation, the position of the perforating branches of the inferior abdominal artery, internal thoracic artery, and dorsal thoracic artery were measured with a Doppler flow detector (Canon Aplio 900, Japan). The flap was designed to be fusiform, with the upper boundary located at the umbilical level and the lower boundary determined according to the degree of skin relaxation. On the premise of satisfying the requirement of covering the chest wall defect as much as possible, both ends of the flap should reach to the axillary front as far as possible and the donor area should be closed. The maximum flap size was $13 \mathrm{~cm} \times 37 \mathrm{~cm}$, and the minimum flap size was $10 \mathrm{~cm} \times 29 \mathrm{~cm}(5)$.

\section{Surgical method}

\section{Resection of the tumor and repair of chest wall}

An area measuring approximately $13 \mathrm{~cm} \times 10 \mathrm{~cm}$ was drawn around the mass of the chest wall, including the reddened skin. The surgeon incised the skin, isolated the subcutaneous fascia, and subcutaneously excised the entire mass along with the pectoralis major muscles and the second and third ribs. The surgeon continued to separate the lower flap until it connected with the abdominal surgical area. The chest wall defect was repaired with 4 layers of ventral hernia patches.

\section{Separation of abdominal flaps}

The anterior superior iliac spine was taken as the horizontal line, the umbilicus was taken as the upper apex of the transverse incision, the apex of the lower line was taken as upper transverse finger of the symphysis pubis, and the fusiform incision was made to separate the subcutaneous tissue. The transverse rectus abdominis myocutaneous flap was separated from the lower abdomen, and the abdominal wall was then repaired. According to the incision designed before the operation, the upper margin of the rectus abdominis myocutaneous flap was separated up to the anterior sheath of the rectus abdominis. The surface of the anterior sheath was separated upward to the rectus abdominis, and across the costal arch to communicate with the thoracic tunnel. The lower margin of the transverse rectus abdominis myocutaneous flap was cut to the anterior sheath of the rectus abdominis. The flap was isolated from the myofascial surface on the contralateral side of the vessel pedicle. The branches perforating the inferior epigastric artery on the right side of the umbilicus should be located preoperatively and protected. The inferior epigastric artery was separated retrograde along the perforation of the abdominal wall and closed with a vascular clamp. The surgeon continued to separate the flap to the middle of the abdomen. The vessel was separated ipsilaterally at the vascular pedicle until the cutaneous vascular perforation at the lateral margin of the rectus abdominis was exposed. A transverse incision was made at the lower edge of the flap to open the anterior sheath of the rectus abdominis, and the inferior abdominal artery and vein were cut off. Another transverse incision was made at the upper edge of the flap to cut the anterior sheath of the rectus abdominis muscle, and the rectus abdominis muscle was separated upward to the costal arch margin to confirm that the arteries and veins on the abdominal wall entered the TRAM flap.

\section{Flap transfer and fixation}

The flap was rotated to the fore chest through subcutaneous tunneling and temporarily fixed. This operation specifically requires the perforator vessels of the flap to be protected. The thoraco-acromion artery and vein were separated by fixing the microscope. During the operation, it was found that the thoracoacromial artery had a good pulse, and it was then anastomosed with the perforating branch of the inferior epigastric artery. Due to the poor condition of the thoracoacromial vein and reflux stasis, the inferior epigastric vein was anastomosed with the axillary vein directly. No leakage was observed at the anastomotic end of the blood vessels, and the blood supply to the flap was improved. The patient's body position was adjusted to the half-sitting position. The abdominal wall was pulled down, and skin holes were made in the upper abdomen. The umbilicus was pulled out through the skin holes and sutured layer by layer 


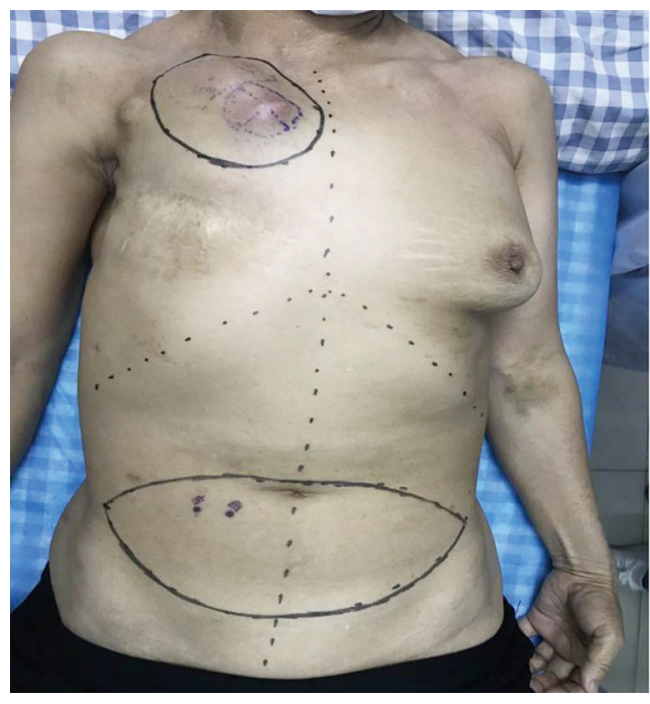

Figure 2 Pre-operation marking line.

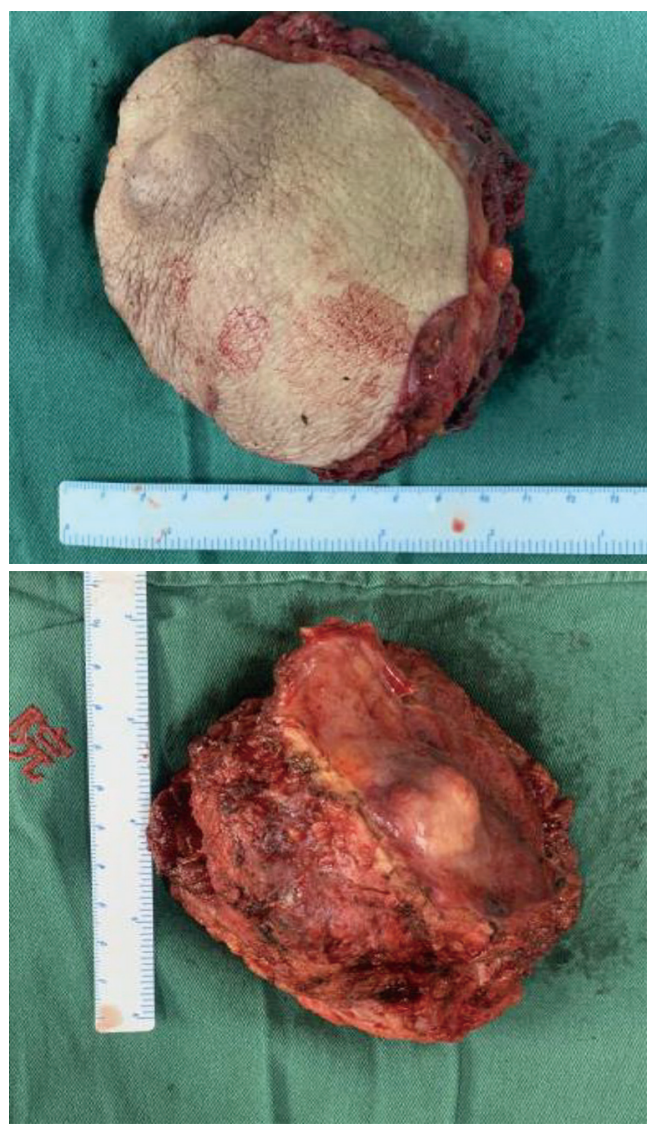

Figure 3 Intraoperative resection of the chest wall mass $(10.5 \mathrm{~cm} \times$ $9.5 \mathrm{~cm})$. to form a new umbilicus. The anterior sheath of the rectus abdominis was sutured, and the lower defect was repaired with a ventral hernia patch to prevent the formation of postoperative ventral hernia. A drainage tube was placed on each side of the bilateral abdominal incision. The abdominal incision was closed in layers, the myocutaneous flap position was adjusted, the myocutaneous flap was trimmed, the redundant flap tissue was cut off, and the upper end and the outer side of the flap were sutured intermittently and fixed to the chest wall. Drainage tubes were placed under the axilla and chest wall, and the incision was sutured (Figures 2-8).

The case and picture were published with the consent of the patient.

\section{Discussion}

With increasingly close collaboration between multiple disciplines (including breast, thoracic, and general surgery), as well as the development of specialized breast plastic surgery and improvements in microscopic reconstruction technology, the repair of complex chest wall defects has achieved more satisfactory results (6). This progress gives patients with local chest wall recurrence the opportunity to undergo radical treatment. For the repair and reconstruction of complex and large chest wall defects resulting from chest wall tumor resection, including bone chest wall and skin soft tissue defects, materials such as bone cement and titanium alloy can be used (7). In the case presented here, only 2 ribs were removed, and the defect scope was not large. A 4-layer high-density polyethylene mesh was used to repair the defect (8), and postoperatively, the patient's chest wall was stable. In clinic, safe and reliable skin flap surgery is used to repair the skin and soft tissue of the chest wall defect. The traditional TRAM flap procedure is simple and can be performed in a short period of time, but the blood supply is not ideal. In this case, the chest wall defect was located at a high position, which required the use of zone 4 of the TRAM flap. Zone 4 of the TRAM flap has the worst blood supply; however, a contralateral DIEP flap can be utilized in combination with TRAM to guarantee the blood supply of the entire flap. This method greatly improved the success rate of the operation. Because the tumor had invaded the blood supply in the thoracic cage, the internal thoracic artery and vein could not be used as 

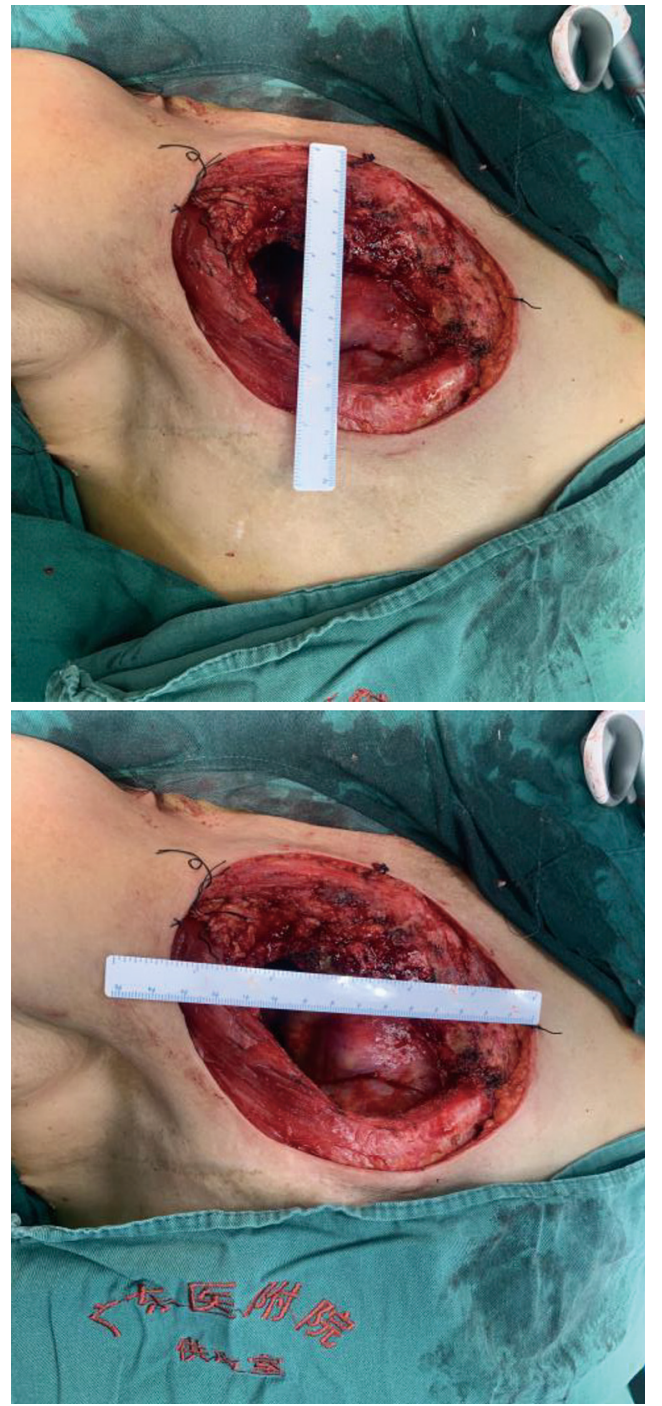

Figure 4 Area of the skin defect after resection of the chest wall mass $(13 \mathrm{~cm} \times 13 \mathrm{~cm})$.

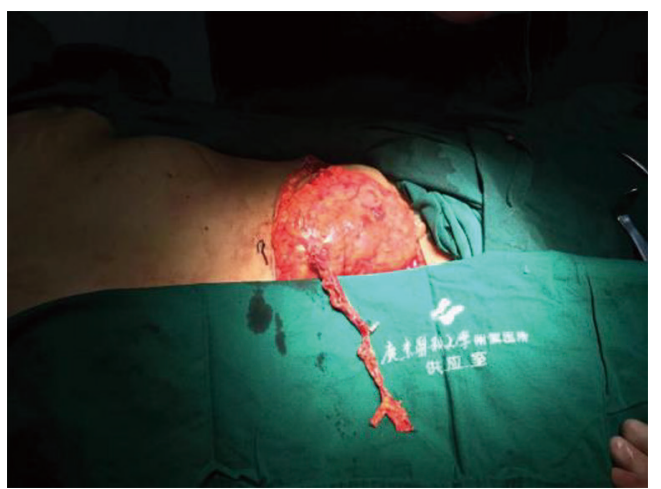

Figure 5 Intraoperative free perforating vessels of the lower abdominal wall.

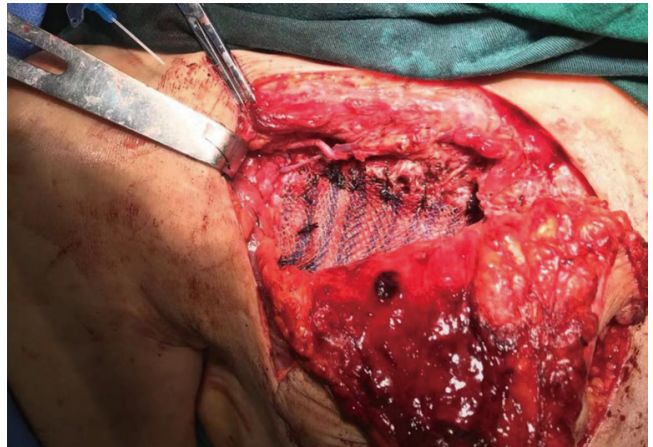

Figure 6 The reconstruction of the chest wall. (I) Repair of the costal defect with a 4-layer mesh; (II) the thoracoacromial artery was anastomosed with inferior epigastric artery perforation (endto-end anastomosis); (III) the inferior epigastric vein perforation was anastomosed with the axillary vein (end-to-side anastomosis).

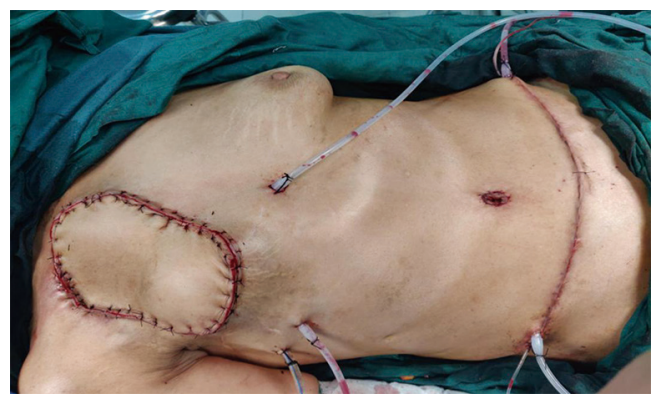

Figure 7 Immediate observation on the completion of flap transplantation during the operation.

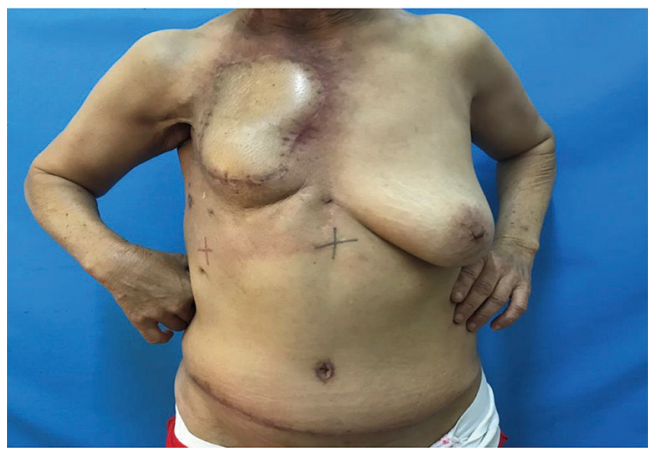

Figure 8 The patient recovered three months after the operation. 
the vessels in the receiving region, so the thoracoacromial artery was selected as the vessel in the receiving region. However, due to the poor condition of thoracoacromial vein and reflux stasis, the inferior abdominal vein was anastomosed with the axillary vein directly at the end (9). At present, research found (10) that after systematic treatment of LABC, the median survival increased from 20 to 50 months and the risk of recurrence ranges from $6 \%$ to $28 \%$. Two years after Systemic therapy, we followed up the patient and found no recurrence of tumor or deformation of chest wall. Most importantly, the mesh did not cause symptoms such as: chest wall pain. Through this method of treatment, the patients' quality of life and mental health have been greatly improved.

\section{Conclusions}

About this method, the soft mesh has a satisfactory robustness; aseptic inflammatory response is small, and in a short period of time, human tissue can grow and cover the mesh network to eliminate the dead cavity (11), which are conducive to wound repair. During surgery, it is easy to cut and use. However, compared with titanium alloy steel plate, its hardness and supporting force is not very satisfactory (12). Sometimes, as the patient breathes, the fixation stitches become loose and the chest wall becomes deformed. This is also the weakness of soft mesh. In the aspect of perforator vascular anastomosis of flap, we need to prepare a number of plans before surgery to prevent poor blood flow in the flap.

In this case, TRAM combined with DIEP was adopted for the chest skin defect through the rational design of the flap. Compared with the traditional TRAM flap, the DIEP flap not only has the same advantages of large tissue volume and easy shaping $(13,14)$, but is also able to overcome the disadvantage of sacrificing part of the rectus abdominis when dissociating the TRAM flap. In this case, when the DIEP flap was removed, the rectus abdominis and its anterior sheath, along with the motor nerves of the related muscles, could be completely retained. This not only ensures the good blood flow of the flap, but also ensures the intact function of the rectus abdominis in the donor area $(15,16)$. The chest wall was repaired with high-density polyethylene mesh, which could effectively rebuild the stability of chest wall. In this case mesh repair plus TRAM combined with DIEP was used reasonably and flexibly for chest wall repair to minimize surgical damage, while multidisciplinary cooperation was helpful in improving the effect of complex chest wall defect repair.

\section{Acknowledgments}

Funding: None.

\section{Footnote}

Reporting Checklist: The authors have completed the CARE reporting checklist. Available at http://dx. doi. org/10. 21037/gs-20-436

Conflicts of Interest: All authors have completed the ICMJE uniform disclosure form (available at http://dx. doi. org/10. 21037/gs-20-436). The authors have no conflicts of interest to declare.

Ethical Statement: The authors are accountable for all aspects of the work in ensuring that questions related to the accuracy or integrity of any part of the work are appropriately investigated and resolved. All procedures performed in studies involving human participants were in accordance with the Declaration of Helsinki (as revised in 2013). The case and picture were published with the consent of the patient.

Open Access Statement: This is an Open Access article distributed in accordance with the Creative Commons Attribution-NonCommercial-NoDerivs 4.0 International License (CC BY-NC-ND 4.0), which permits the noncommercial replication and distribution of the article with the strict proviso that no changes or edits are made and the original work is properly cited (including links to both the formal publication through the relevant DOI and the license). See: https://creativecommons.org/licenses/by-nc-nd/4.0/.

\section{References}

1. Tryfonidis K, Senkus E, Cardoso MJ, et al. Management of locally advanced breast cancer-perspectives and future directions. Nat Rev Clin Oncol 2015;12:147-62.

2. Qi Y, Li X, Zhao S, et al. Value of porous titanium alloy plates for chest wall reconstruction after resection of chest wall tumors. Asian Pac J Cancer Prev 2014;15:4535-8.

3. Tamburini N, Grossi W, Sanna S, et al. Chest wall reconstruction using a new titanium mesh: a multicenters experience. J Thorac Dis 2019;11:3459-66.

4. Sandler G, Hayes-Jordan A. Chest wall reconstruction after tumor resection. Semin Pediatr Surg 2018;27:200-6.

5. Gupta AK, Aggarwal R, Kumar S, et al. Prospective study 
on Clinico-Pathological profile and treatment outcome in locally advanced breast cancers (LABC). International Journal of Contemporary Surgery 2018;6:69-74.

6. Luan J, Mu L, Mu L, et al. Comparison of DIEPTRAM combined flap and bilateral DIEP flap breast reconstruction. Journal of Tissue Engineering and Reconstruction Surgery 2006;(05):267-9.

7. Song D, Li Z, Zhou X, et al. Repair and reconstruction of postoperative defects of chest wall tumors. Chinese Clinical Medicine 2017;24:112 $\leq 11-5$.

8. Lardinois D, Müller M, Furrer M, et al. Functional assessment of chest wall integrity after methylmethacrylate reconstruction. Ann Thorac Surg 2000;69:919-23.

9. Chen K, Yu P. Chest wall resection and reconstruction. Chinese Journal of Surgery 2005;43:955-8.

10. Arce K, Bell RB, Potter JK, et al. Vascularized free tissue transfer for reconstruction of ablative defects in oral and oropharyngeal cancer patients undergoing salvage surgery following concomitant chemoradiation. Int J Oral Maxillofac Surg 2012;41:733-8.

Cite this article as: Huang S, Qiu P, Zhang Y, Li J, Chen W, Chen B, Liang Z, Liang Z, Luo K, Huang B. Reconstruction of the chest wall in locally advanced breast cancer with multidisciplinary cooperation: a case report of mesh repair plus TRAM combined with DIEP chest wall reconstruction. Gland Surg 2020;9(4):1048-1055. doi: 10.21037/gs-20-436
11. Du R, Yu N, Long X, et al. Application progress of chest wall reconstruction materials. Journal of Chinese Academy of Medical Sciences 2008;40:289-93.

12. Koole D, Bemelman M, Schijen J, et al. Reconstruction of chest wall defects using a technique involving mesh, titanium plates, and a pedunculated muscle flap. Korean J Thorac Cardiovasc Surg 2018;51:308-11.

13. Parker SJ, Harries SA. Phyllodes tumours. Postgrad Med J 2001;77:428-35.

14. Lindenblatt N, Gruenherz L, Farhadi J. A systematic review of donor site aesthetic and complications after deep inferior epigastric perforator flap breast reconstruction. Gland Surg 2019;8:389-98.

15. Allen RJ. DIEP versus TRAM for breast reconstruction. Plast Reconstr Surg 2003;111:2478.

16. $\mathrm{Xu} \mathrm{J}, \mathrm{Mu} \mathrm{O}$, Liu Y, et al. Application of perforating branch flap of inferior abdominal artery in breast reconstruction and repair of chest wall ulcer. Chinese Journal of Surgery 2001;04:49-51. 